\title{
Extracellular vesicles can be processed by electrospinning without loss of structure or function
}

\author{
Rita Pereira Trindade, ${ }^{\mathrm{a}}$ Nisa Renault, ${ }^{\mathrm{b}}$ Nadia El Harane, ${ }^{\mathrm{c}}$ Philippe Menaschéd and Gareth R. Williams*a \\ ${ }^{a}$ UCL School of Pharmacy, University College London, 29-39 Brunswick Square, London, WC1N 1AX, \\ UK. \\ ${ }^{\mathrm{b}}$ Fujifilm Cellular Dynamics Inc., 525 Science Drive, Madison, Wisconsin, 53711, USA. \\ c Université de Paris, PARCC, INSERM, F-75015 Paris, France. \\ ${ }^{d}$ Department of Cardiovascular Surgery, Hôpital Européen Georges, Université de Paris, PARCC, \\ INSERM, F-75015 Paris, France.
}

\begin{abstract}
Extracellular vesicles (EVs) are cell-derived bodies proven to have a wide range of therapeutic applications. To date, EVs have almost always been administered by direct injection, which is very likely to hinder their efficacy because of rapid clearance from the injection site. Here we show that EVs can be successfully processed into polymer-based fibres by electrospinning, with no loss of structure or function.
\end{abstract}

\section{Keywords}

Electrospinning, nanofibres, extracellular vesicles, tissue regeneration

\section{Introduction}

Proteins, genetic material, antibodies and now even cells are commercially used as active ingredients in medical interventions. Such biopharmaceuticals benefit from high specificity and potency compared to small molecule drugs, but their structural complexity poses extra formulation and delivery challenges, especially regarding stability.

Extracellular vesicles (EVs) are anuclear vesicles bounded by a lipid bilayer membrane. They are released by many different cells and play pivotal roles in both physiological and pathological conditions [1, 2]. EVs have great potential as diagnostic and therapeutic tools, particularly in tissue regeneration: they have for instance been found to protect the heart from ischemia $[3,4]$ and aid cardiac repair after myocardial infarction [5]. EVs are less immunogenic $[6]$ and tumorigenic $[7,8]$ than the stem cells which are usually employed for tissue engineering, and have been shown to be equally as effective in promoting regeneration of cardiac tissue [9]. EVs further permit greater dosing reproducibility [10] and their production is easier to scale up [6]. They thus have enormous therapeutic potential, but almost all studies exploring this to date have used direct injection of EV suspensions as the delivery route. This is both invasive and sub-optimal, because the EV suspension is likely to be rapidly cleared from the target area if injected in a snap-shot fashion. This will temper their potential therapeutic benefits. There exists a clear need for more sophisticated formulation strategies for EV 
delivery in order to fully maximise their benefit to patients. So far, there is only one report of such a strategy having been employed, using a hydrogel patch [11].

One strategy which has been shown to be very promising for the processing of biologics is electrospinning, which employs electrical energy to convert a polymer solution containing a functional component into solid nanoscale fibres [12]. DNA [13], proteins (e.g. bevacizumab [14]), and even cells [15] can all be electrospun without significant loss of their integrity or functional performance. Jayasinghe et al. [15] have shown that the cells in electrospun fibres remain viable and can be used for a range of therapeutic applications. In this work, we sought to determine whether EVs can be processed by electrospinning, and if so what influence this has on their properties. We opted to generate fast-dissolving materials as a proof-of-concept, since this allowed us to immediately assay structure and functional performance after fabrication.

\section{Methods}

A polyvinylpyrrolidone (PVP; Mw $360 \mathrm{kDa}$ ) solution in trifluoroethane was prepared at $10 \% \mathrm{w} / \mathrm{v}$. EVs were isolated from foetal bovine serum (FBS) and mesenchymal stem cell (MSC) media using ultracentrifugation and suspended in phosphate buffered saline (PBS). The EV suspension was added to the PVP solution to give a final EV concentration of $3.75 \times 10^{8}$ particles $/ \mathrm{mL}$. Electrospinning was performed at a flow rate of $1 \mathrm{~mL} / \mathrm{h}$, a spinneret-to-collector distance of $18 \mathrm{~cm}$ and an applied voltage of $8 \mathrm{kV}$. The fibres produced were analysed by scanning electron microscopy (SEM), and dissolved for additional analysis by nanoparticle tracking analysis (NTA), transmission electron microscopy (TEM), Bradford assays, and in cell culture. Full details are given in the Supplementary Information.

\section{Results and discussion}

SEM images (Fig. 1) revealed that smooth fibres were obtained for both blank and EV-loaded formulations. A reduction in fibre diameter occurs upon the addition of EVs, because of the increase in conductivity which arises when adding EVs to the PVP solution (see Fig. S1, Supporting Information).
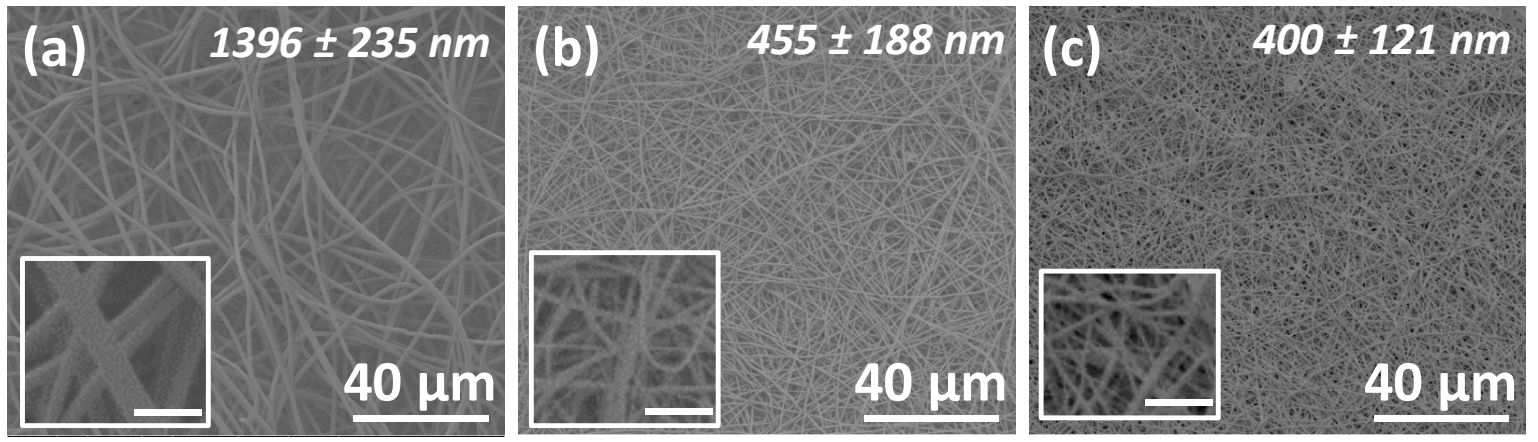

Fig. 1. SEM images of (a) blank PVP; (b) PVP-FBS EV; and, (c) PVP-MSC EV fibres. Inset scale bar: $5 \mu \mathrm{m}$.

To explore the extent of EV encapsulation in the polymer matrix, NTA was performed on fibres dissolved in PBS (Fig. S2). It was not possible to directly detect the formulated EVs due to the noise arising from the polymer. However, there is a distinct difference in the overall concentration of particles detected. NTA data on the blank PVP fibres revealed a concentration of $1.03 \times 10^{9} \pm 6.36 \mathrm{x}$ 
$10^{7}$ particles $/ \mathrm{mL}$, a $4.0 \times 10^{8}$ difference from the $1.43 \times 10^{9} \pm 5.19 \times 10^{7}$ particles $/ \mathrm{mL}$ obtained with the FBS EV-loaded formulation. This difference is close to the theoretical EV loading of $3.75 \times 10^{8} \mathrm{EVs} / \mathrm{mL}$.

TEM (Fig. 2) confirmed the presence of EVs after the fibres had been dissolved. Spherical structures of $50 \pm 10 \mathrm{~nm}$ with a stained lipid bilayer can clearly be seen, consistent with the literature [16].

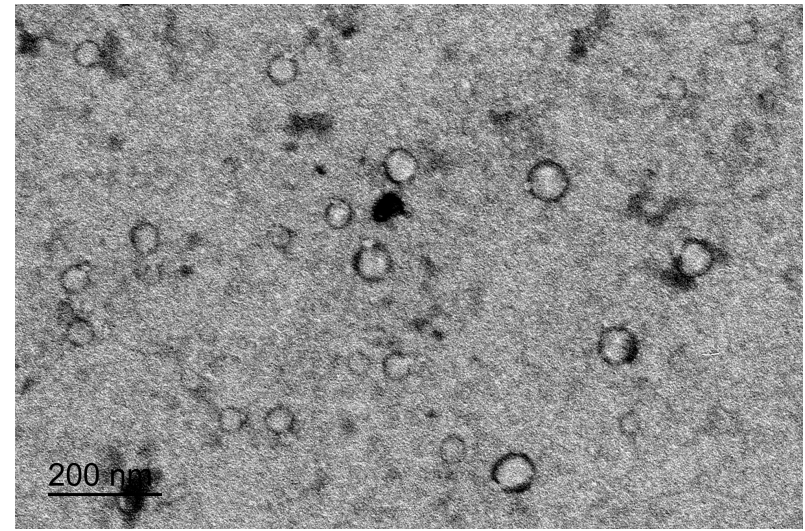

Fig. 2. TEM images of dissolved PVP-FBS EV fibres.

EV incorporation into the fibres was also assessed in terms of protein content (Fig. S3). The total protein content was lower for the formulated samples than with the unprocessed EV. The encapsulation efficiency is estimated at $76.8 \pm 1.6 \%$ for the FBS EV-loaded fibres and $70.6 \pm 8.5 \%$ for the MSC EVs system. This high encapsulation efficiency is very promising for onward applications.

EV structure and protein content are clearly retained after electrospinning. It is also crucial to understand whether the formulated vesicles are still potent post-processing. To determine their potency, stress survival assays were performed using rat cardiomyocytes (Fig. 3).

(a)

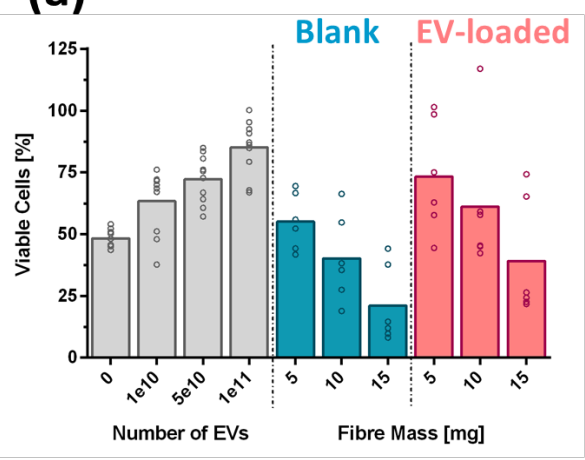

(b)

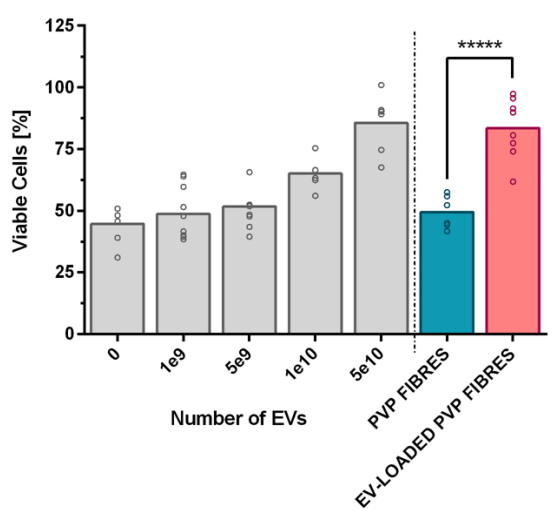

Fig. 3. Cell viability data for (a) PVP-FBS EV and (b) PVP-MSC EV fibres. Data are shown as mean \pm S.D. $(\mathrm{n}=3$ ). $* * * * * \mathrm{p}<$ 0.0001. The fibre masses in (a) correspond to the following theoretical EV numbers: $5 \mathrm{mg} \mathrm{ca} .1 .88 \times 10^{10}, 10 \mathrm{mg} c a .3 .75 \times 10^{10}$ and $15 \mathrm{mg} \mathrm{ca} .5 .63 \times 10^{10}$ particles. In (b), $5 \mathrm{mg}$ of fibres was added, giving $1.88 \times 10^{10} \mathrm{EVs}$.

The percentage of viable cells increases after the addition of FBS EVs, regardless of whether the vesicles were formulated or not. Adding $5 \mathrm{mg}$ of FBS EV-loaded fibres produced a survival after stress of $73 \pm 21 \%$, equivalent to the viability upon addition of the same number of unprocessed EVs. At this concentration, the polymer added does not seem to influence viability. Surprisingly, as the amount of FBS EV-loaded fibres added is increased there is a decrease in cell survival (Fig. 3a). This arises because the PVP is detrimental to cardiomyocyte survival, owing to the relatively high ratio of polymer mass per surface area and total volume of media in the well. The observed decline in viability with adding 
more FBS EV fibres is of the same order of magnitude as seen with the blank PVP fibres. It appears that the positive effect of the additional number of EVs present and the detrimental effect of the increased PVP content balance out to give a net negative effect. Overall however, it is clear that FBS EVs can be electrospun and still remain as potent as before processing.

The addition of MSC EV-loaded fibres produced a survival after stress of $87 \pm 9 \%$ (Fig. 3b), equivalent to the value found upon treatment with the same number of unprocessed EVs. Mesenchymal stem cell derived EVs can thus also be electrospun with no loss in potency. These results highlight the feasibility of using electrospinning as an effective formulation strategy for EVs. This opens up the possibilities of storing EVs in a solid medium ready for reconstitution for injection, for example, or to apply topically directly.

\section{Conclusions}

Here we have shown that EVs can be easily processed by electrospinning, without losing their structure or potency. In the longer term, the production of EV-loaded fibres based on slow release polymers would enable extended release of EVs in vivo, providing the cues required for tissue regeneration over a prolonged period of time and ultimately resulting in improved tissue regeneration (e.g. after cardiac ischaemia).

\section{Acknowledgements}

The authors would like to thank the Engineering and Physical Sciences Research Council (EPSRC) for PhD funding to RPT through the Centre for Doctoral Training in Advanced Therapeutics \& Nanomedicines (EP/L01646X/1). Funding was provided to INSERM from LabEx REVIVE (ANR-10-LABX73); the Fondation pour la Recherche Médicale (DEQ20160334910); and the Fondation de France (FDF/2014 00047970).

\section{References}

[1] Y. Lee, S. EL Andaloussi, M.J.A. Wood, Exosomes and microvesicles: extracellular vesicles for genetic information transfer and gene therapy, Human Molecular Genetics 21(R1) (2012) R125-R134.

[2] H. Valadi, K. Ekström, A. Bossios, M. Sjöstrand, J.J. Lee, J.O. Lötvall, Exosome-mediated transfer of mRNAs and microRNAs is a novel mechanism of genetic exchange between cells, Nature Cell Biology 9 (2007) 654.

[3] L. Chen, Y. Wang, Y. Pan, L. Zhang, C. Shen, G. Qin, M. Ashraf, N. Weintraub, G. Ma, Y. Tang, Cardiac progenitor-derived exosomes protect ischemic myocardium from acute ischemia/reperfusion injury, Biochemical and Biophysics Research Communications 431(3) (2013) 566-71.

[4] Z. Giricz, Z.V. Varga, T. Baranyai, P. Sipos, K. Paloczi, A. Kittel, E.I. Buzas, P. Ferdinandy, Cardioprotection by remote ischemic preconditioning of the rat heart is mediated by extracellular vesicles, Journal of Molecular Cell Cardiology 68 (2014) 75-8.

[5] S. Sahoo, D.W. Losordo, Exosomes and cardiac repair after myocardial infarction, Circulation Research 114(2) (2014) 333-44.

[6] T.S. Chen, F. Arslan, Y. Yin, S.S. Tan, R.C. Lai, A.B. Choo, J. Padmanabhan, C.N. Lee, D.P. de Kleijn, S.K. Lim, Enabling a robust scalable manufacturing process for therapeutic exosomes through oncogenic immortalization of human ESC-derived MSCs, Journal of Translational Medicine 9 (2011) 47. 
[7] H. Yamakawa, M. leda, Strategies for heart regeneration, International Heart Journal 56(1) (2015) 1-5.

[8] L. Zhang, Y. Pan, G. Qin, L. Chen, T.K. Chatterjee, N.L. Weintraub, Y. Tang, Inhibition of stearoyl-coA desaturase selectively eliminates tumorigenic Nanog-positive cells: improving the safety of iPS cell transplantation to myocardium, Cell Cycle 13(5) (2014) 762-71.

[9] A. Kervadec, V. Bellamy, N. El Harane, L. Arakélian, V. Vanneaux, I. Cacciapuoti, H. Nemetalla, M.C. Périer, H.D. Toeg, A. Richart, M. Lemitre, M. Yin, X. Loyer, J. Larghero, A. Hagège, M. Ruel, C.M. Boulanger, J.-S. Silvestre, P. Menasché, N.K.E. Renault, Cardiovascular progenitor-derived extracellular vesicles recapitulate the beneficial effects of their parent cells in the treatment of chronic heart failure, Journal of Heart and Lung Transplantation 35(6) (2016) 795-807.

[10] G. Maguire, Stem cell therapy without the cells, Commun. Integrated Biology 6(6) (2013) e26631. [11] B. Liu, B.W. Lee, K. Nakanishi, A. Villasante, R. Williamson, J. Metz, J. Kim, M. Kanai, L. Bi, K. Brown, G. Di Paolo, S. Homma, P.A. Sims, V.K. Topkara, G. Vunjak-Novakovic, Cardiac recovery via extended cell-free delivery of extracellular vesicles secreted by cardiomyocytes derived from induced pluripotent stem cells, Nature Biomedical Engineering 2(5) (2018) 293-303.

[12] G.R. Williams, B.T. Raimi-Abraham, C.J. Luo, Nanofibres in drug delivery, UCL Press, London, 2018. [13] Y.K. Luu, K. Kim, B.S. Hsiao, B. Chu, M. Hadjiargyrou, Development of a nanostructured DNA delivery scaffold via electrospinning of PLGA and PLA-PEG block copolymers, Journal of Controlled Release 89(2) (2003) 341-353.

[14] U. Angkawinitwong, S. Awwad, P.T. Khaw, S. Brocchini, G.R. Williams, Electrospun formulations of bevacizumab for sustained release in the eye, Acta Biomaterialia 64 (2017) 126-136.

[15] A. Townsend-Nicholson, S.N. Jayasinghe, Cell Electrospinning: a Unique Biotechnique for Encapsulating Living Organisms for Generating Active Biological Microthreads/Scaffolds, Biomacromolecules 7(12) (2006) 3364-3369.

[16] X. Loyer, I. Zlatanova, C. Devue, M. Yin, K.Y. Howangyin, P. Klaihmon, C.L. Guerin, M. Kheloufi, J. Vilar, K. Zannis, B.K. Fleischmann, D.W. Hwang, J. Park, H. Lee, P. Menasche, J.S. Silvestre, C.M. Boulanger, Intra-Cardiac Release of Extracellular Vesicles Shapes Inflammation Following Myocardial Infarction, Circulation Research 123(1) (2018) 100-106.

[17] C. Théry, S. Amigorena, G. Raposo, A. Clayton, Isolation and Characterization of Exosomes from Cell Culture Supernatants and Biological Fluids, Current Protocols in Cell Biology 30(1) (2006) 3.22.13.22.29. 